\title{
Conduction Velocity of Action Potentials Measured from Unidimensional Latency-topography in Human and Frog Skeletal Muscle Fibers
}

\author{
Saburo Homma, Yoshio Nakajima, Kenji Hayashi, * \\ and Shinobu Toma \\ Department of Physiology, School of Medicine, \\ Chiba University, Chiba, 280 Japan \\ *Department of Orthopaedic Surgery, Chiba Rosai Hospital, \\ Ichihara, 229 Japan
}

\begin{abstract}
Conduction of an action potential along skeletal muscle fibers was graphically displayed by unidimensional latency-topography, UDLT. Since the slopes of the equipotential line were linear and the width of the line was constant, it was possible to calculate conduction velocity from the slope. To determine conduction direction of the muscle action potential elicited by electric stimulation applied directly to the muscle, surface recording electrodes were placed on a two-dimensional plane over a human muscle. Thus a bi-dimensional topography was obtained. Then, twelve or sixteen surface electrodes were placed linearly along the longitudinal direction of the action potential conduction which was disclosed by the bi-dimensional topography. Thus conduction velocity of muscle action potential in man, calculated from the slope, was for $\mathrm{m}$. brachioradialis, $3.9 \pm 0.4 \mathrm{~m} / \mathrm{s}$; for $\mathrm{m}$. biceps brachii, $3.6 \pm 0.2 \mathrm{~m} / \mathrm{s}$; for $\mathrm{m}$. sternocleidomastoideus, $3.6 \pm 0.4 \mathrm{~m} / \mathrm{s}$. By using a tungsten microelectrode to stimulate the motor axons, a convex-like equipotential line of an action potential in UDLT was obtained from human muscle fibers. Since a similar pattern of UDLT was obtained from experiments on isolated frog muscles, in which the muscle action potential was elicited by stimulating the motor axon, it was assumed that the maximum of the curve corresponds to the end-plate region, and that the slopes on both sides indicate bi-directional conduction of the action potential.
\end{abstract}

Key words: conduction velocity, human muscle fiber, unidimensional latency-topography.

We developed a computer-aided method, by which the conduction of an impulse along an excitable membrane can be graphically shown as a color display. Since recorded action potentials were graphically displayed (abscissa, location of

Received for publication August 23, 1985 
recording electrodes; ordinate, latency; color tint, amplitude), we called this graphic display unidimensional latency-topography, UDLT (Homma et al., 1983a).

Application of this method to an action potential, elicited by intracellularly applied current to a frog skeletal muscle fiber, revealed a linear equipotential relation between the electrode position $(\mathrm{mm})$ and the latency (ms) (Homma et al., 1983a). Conduction of an action potential along the muscle fiber was continuous. This method was also applied to impulse conduction in rat spinal root fibers, in which the recording electrodes were placed at very short intervals to match the internodal length of the nerve fiber; the calculated UDLT showed a step-like relation (Hомма et al., 1983b, 1984). It was confirmed that the UDLT showed saltatory conduction of an impulse when compared with the length-latency relationship obtained by other methods (HUXLEY and STÄMPFLI, 1949; RASMINSKY and SEARS, 1972).

In the present investigation, we have applied this method to impulse conduction along human skeletal muscle fibers in order to measure the conduction velocity of action potentials elicited by electric stimulation applied to the muscle through surface electrodes. Furthermore, using the microneurographic technique, action potentials elicited by electric stimulation of motor axons were recorded from surface electrodes placed longitudinally along the muscle fiber. The UDLT showed a convex-like distance-latency relation. To explain the convexity shown in the UDLT, a single motor axon innervating the frog sartorius muscle was stimulated. The UDLT obtained from the frog muscle fiber also showed a convex-like relation. It was assumed that the convex-like distance-latency relationship coincides with the bi-directional conduction of the action potential from the end-plate region.

\section{METHODS}

1. Experiments on human subjects. Experiments were performed on sixteen young, healthy adults. In sections $1 \mathrm{a}$ and $1 \mathrm{~b}$ of the RESULTS, the brachioradialis, biceps brachii and sternocleidomastoid muscles were selected for investigation. Cup-shaped silver electrodes ( $5 \mathrm{~mm}$ diameter) filled with electrolyte paste were used for recording and placed on the skin over the muscle. In section 1a, the recording electrodes were set at sixteen crossing points of an $8 \times 8 \mathrm{~cm}$ matrix at $2 \mathrm{~cm}$ intervals (see the inset diagram in Fig. 1). In section $1 \mathrm{~b}$, twelve electrodes, similar to those mentioned above, were placed in a line on the skin at a $1 \mathrm{~cm}$ interval along the direction of the action potential conduction that was revealed by the electrode arrangement described in the section 1a. The muscle fibers were electrically stimulated with a surface electrode placed proximal to the recording electrodes. The indifferent electrode was set distal to the recording ones, while an earthed cotton cloth was wrapped round the arm between the stimulating and recording electrodes.

In section 1c, a tungsten microelectrode prepared for a microneurogram (tip diameter, $2 \mu \mathrm{m}$; impedance, $10 \mathrm{M} \Omega$ at $1 \mathrm{kHz}$ ) was inserted into the muscle nerve fasciculus of the median nerve at the level of the elbow joint to stimulate motor 
axons innervating the flexor pollicis brevis muscle. Connector pins for an integrated circuit, which were linearly lined up at $5 \mathrm{~mm}$ intervals, were used to record action potentials from the flexor pollicis brevis muscle fiber. An indifferent electrode and an earthed plate were placed on the skin at the elbow joint.

2. Experiments on the frog. The sartorius muscle of the frog (Rana catesbeiana) was isolated along with its innervating nerve fibers and was placed on an electrode array containing sixteen electrodes arranged side by side at $2 \mathrm{~mm}$ intervals. The innervating nerve fibers were finely dissected to permit stimulation of a single motor axon, which was confirmed by the presence of all or none of the muscle action potential. Dantrolene sodium dissolved in the Ringer solution was used to prevent the twitch contraction of the muscle. Details of the experiments on the frog appeared in Homma et al. (1983a).

3. Data acquisition and processing. Muscle action potentials, elicited by electric stimulation to the motor axons or the muscle fibers, were simultaneously sampled and averaged from eight to thirty two times, using a signal processor (7T17, NEC San-ei Ltd. Co.).

Detailed descriptions of the computer interpolation and its topographical display have appeared in previous papers (Homma et al., 1981, 1983a). Briefly, the signal processor interpolated the amplitude gradients of action potentials between the recording electrode positions, and the interpolated amplitudes were displayed in different colors. Since the recording electrodes were placed on a two-dimensional plane in the section la of the RESULTS, the topography was called bi-dimensional topography in this study. But in sections $1 \mathrm{~b}, 1 \mathrm{c}$, and 2 , the recording electrodes were placed linearly and displayed as the abscissa of topography. Therefore this was called unidimensional topography of an action potential. By scanning the unidimensional topography in time (latency), unidimensional latency-topography was obtained. Using the topographic map with latency displayed as the ordinate, distance as the abscissa, and color indicating the amplitude, it is possible to calculate conduction velocity from the slope of an equipotential line displayed with a given color.

\section{RESULTS}

\section{1a. Bi-dimensional topography obtained from direct stimulation of human muscle}

Figure 1 shows an example obtained from the $\mathrm{m}$. brachioradialis. Topographic representations of the action potential, elicited by electric stimulation applied directly to the muscle, are shown at $2 \mathrm{~ms}$ intervals. In this case, stimulus intensity was adjusted to elicit a very small and weak contraction of the muscle, which was just visible under the skin. In Fig. 1, blue and red colors represent negative and positive potentials, respectively. It is apparent from Fig. 1 that the negative wave was generated $10 \mathrm{~ms}$ after the beginning of stimulation under electrode 1 and went straight down from there to electrodes $3,5,7$, and 9, successively. It is also obvious that the positive wave followed the movement of the negative. Figure 1 indicates that 
the action potential moved along the muscle fiber under electrodes 1, 3, 5, 7, and 9 .

Since the conductive direction of the action potentials in the muscle fiber was clearly determined by this experiment, twelve surface electrodes were linearly placed on the skin at $1 \mathrm{~cm}$ intervals coinciding with the conductive direction of the action potentials in the same subject.

\section{1b. UDLT obtained by direct stimulation of muscle}

Action potentials of the brachioradialis muscle, elicited by electrical stimulation applied directly to the muscle, are shown in Fig. 2. It is obvious that the latency of the large negative wave was successively longer in electrodes progressively more distal to the stimulating electrode. The amplitude became smaller as the action potential moved distally. This is probably due to the muscle fibers going deeper from the skin as they run distally.

Unidimensional latency-topography (UDLT) of Fig. 2 is shown in Fig. 3A. It is apparent that the negative wave of Fig. 2 is displayed linearly, which means that the negative wave was conducted continuously. The multiphasic wave of Fig. 2 is shown as several lines of different colors in Fig. 3A. From the first blue line, conduction velocity was calculated to be about $4.0 \mathrm{~m} / \mathrm{s}$. In many examples obtained in this study, a second blue line was observed. This seems to mean that muscle fibers with slow conduction velocity were stimulated. The conduction velocity measured from the second blue line in Fig. 3A was $3.4 \mathrm{~m} / \mathrm{s}$. In the present investigation, only the first blue line was analyzed for the reasons that are described in the discussion.

Figure $3 \mathrm{~B}$ and $\mathrm{C}$ shows the UDLT of impulse conduction in the biceps brachii and sternocleidomastoid muscles, respectively. In each case, negative (blue) and positive (red) waves show a linear relationship between latency (time) and electrode location (distance). Conduction velocity measured from these topographic maps is summarized in Table 1. Conduction velocities obtained from the three muscles had almost the same value. In Table 1, conduction velocity obtained from frog sartorius muscles (Hомма et al., 1983a) is shown for the purpose of comparison. Conduction velocity of human muscle fibers was faster than that of frog muscle fibers.

\section{1c. Stimulation of single motor axons and UDLT of human muscle fiber}

In Fig. 4, a tungsten microelectrode was inserted into a muscle nerve fasciculus of the median nerve. A muscle contraction was elicited by applying current to the motor axons through the microelectrode. By moving the microelectrode manually and reducing the intensity of the current, it was possible to elicit a very weak fascicular contraction of the muscle. In this study, muscle fibers in the $\mathrm{m}$. flexor pollicis brevis were activated.

Twelve surface electrodes were lined up linearly along the length of the thumb, which was determined from the visible fascicular contraction of the muscle, as shown in Fig. 4A. Action potentials were recorded from electrode 1 to 5 as shown in Fig. 4B. Action potentials recorded from electrode 6 to 12 were much smaller than those recorded from electrode 5 and had a quite similar latency as shown in Fig. 3D 


\section{Latency}
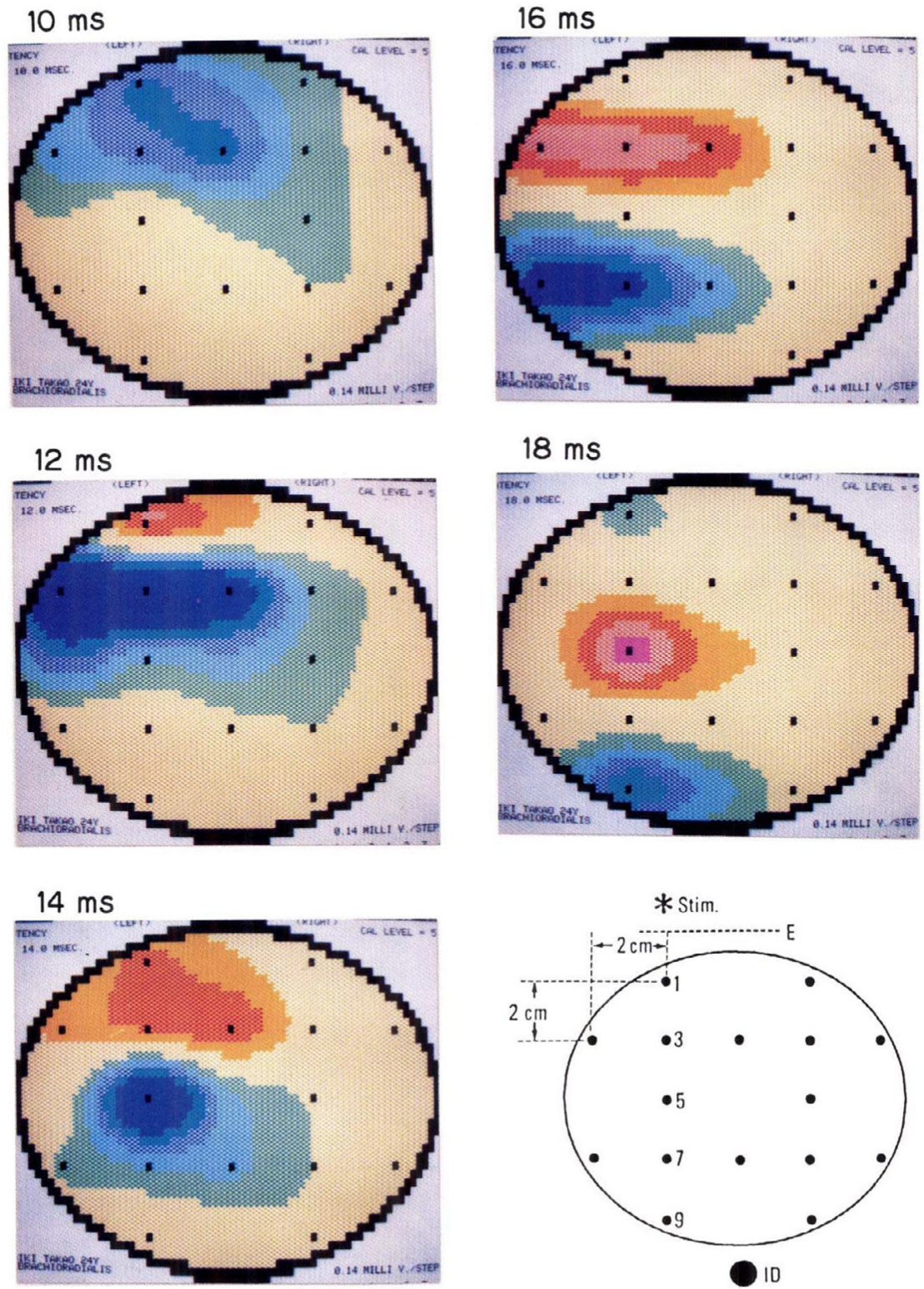

Fig. 1. Bi-dimensional topographies of action potentials in the brachioradialis muscle of man. The topographies were successively obtained from the latencies indicated at the upper left of each topography. On the lower right, the electrode arrangement is schematically shown, and some of the recording electrodes are indicated by numbers. Abbreviations: Stim., stimulating electrode; E, body earth; ID, indifferent electrode. 

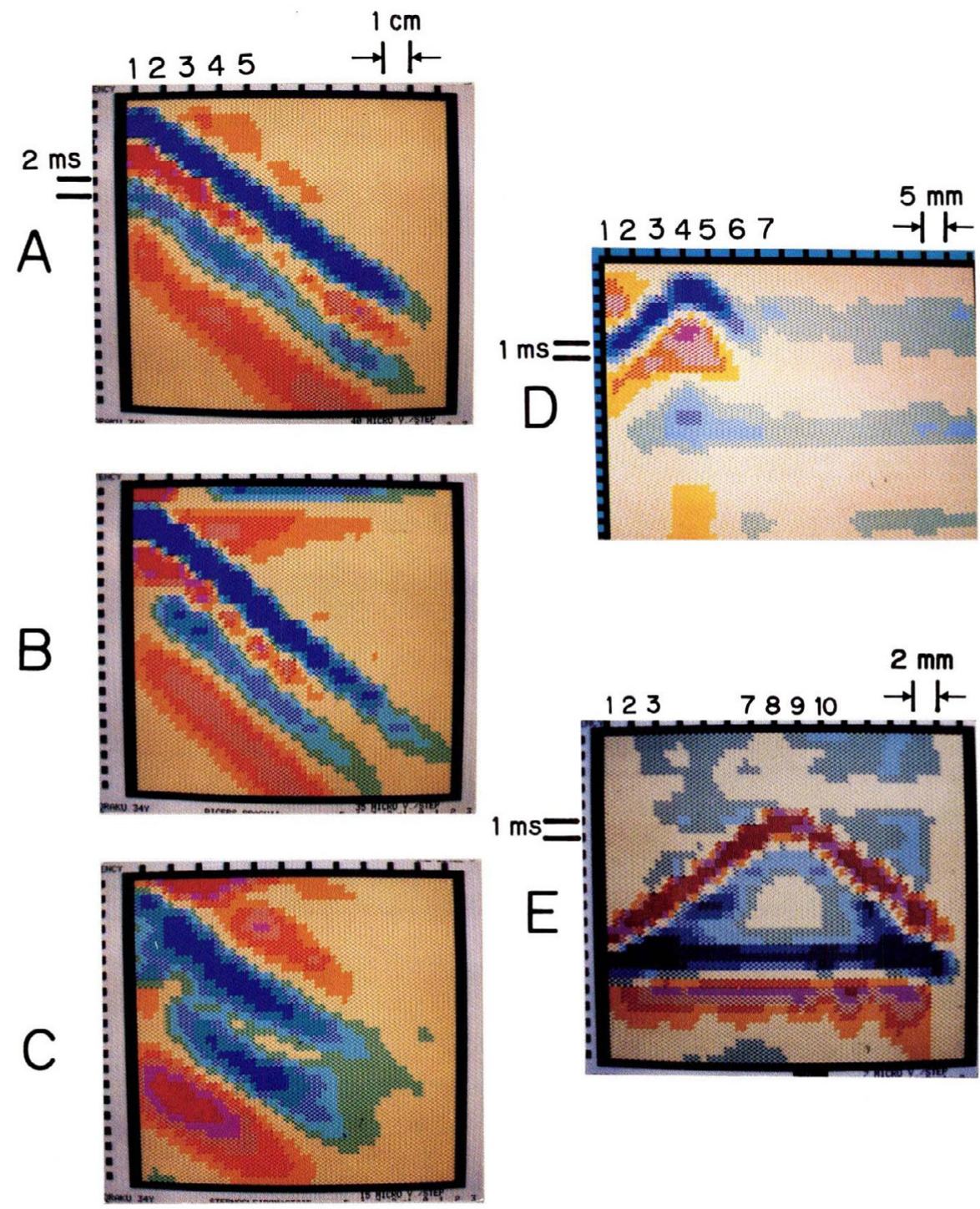

Fig. 3. Unidimensional latency-topographic maps of: A, brachioradialis; B, biceps brachii; C, sternocleidomastoid; D, flexor pollicis brevis muscles in man; E, frog sartorius muscle.

(light blue line), so they were depicted as a horizontal line.

Figure 3D shows the UDLT calculated from the action potentials shown in Fig. 4B. As seen in Fig. 3D, negative potential (deep blue) showed a convex-like slope. Since the distribution pattern of the negative potentials with the up- and downward slopes were similar and linear, it was assumed that the plateau portion 


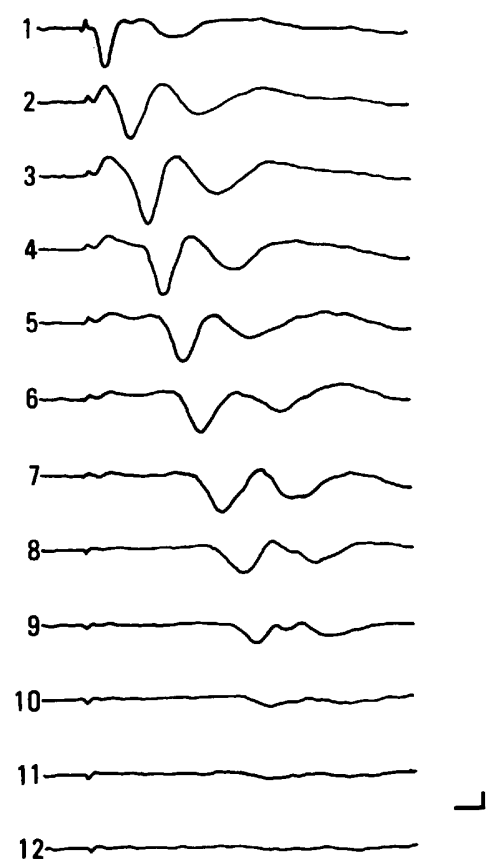

Fig. 2. Twelve action potentials recorded from surface electrodes lined up linearly along the direction of impulse conduction disclosed in Fig. 1. The electrodes were placed at $1 \mathrm{~cm}$ intervals on a line connecting electrodes 1 and 9 . Calibration bars indicate $0.5 \mathrm{mV}$ (upward positive) and $4 \mathrm{~ms}$, respectively.

Table 1. Conduction velocity measured from UDLTs in man.

\begin{tabular}{clcc}
\hline & & $\begin{array}{c}\text { Number of } \\
\text { observations }\end{array}$ & $\begin{array}{c}\text { C.V. (m/s) } \\
(\text { mean } \pm \text { S.D. })\end{array}$ \\
\hline Frog* & Sartorius & 29 & $1.9 \pm 0.3\left(T=20^{\circ} \mathrm{C}\right)$ \\
\hline \multirow{2}{*}{ Man } & Brachioradialis & 16 & $3.9 \pm 0.4$ \\
& Biceps brachii & 16 & $3.6 \pm 0.2$ \\
& Sternocleidomastoideus & 16 & $3.6 \pm 0.4$ \\
\hline
\end{tabular}

* Cited from Homma et al. (1983a).

under electrode 4 corresponds with the end-plate region of the stimulated motor axon, and that the action potential was conducted bi-directionally and uniformly along muscle fibers belonging to the same motor unit. Whereas the slope was deep blue in color, the conduction velocity was measured to be $3.9 \mathrm{~m} / \mathrm{s}$.

To confirm the assumption that the plateau portion of Fig. 3D corresponds with the end-plate, experiments were performed on the frog muscle fiber. 
A

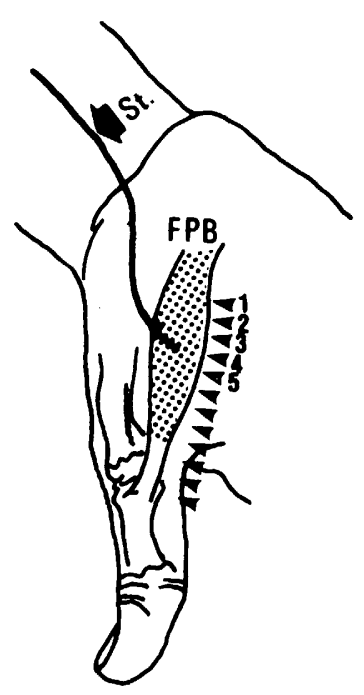

B

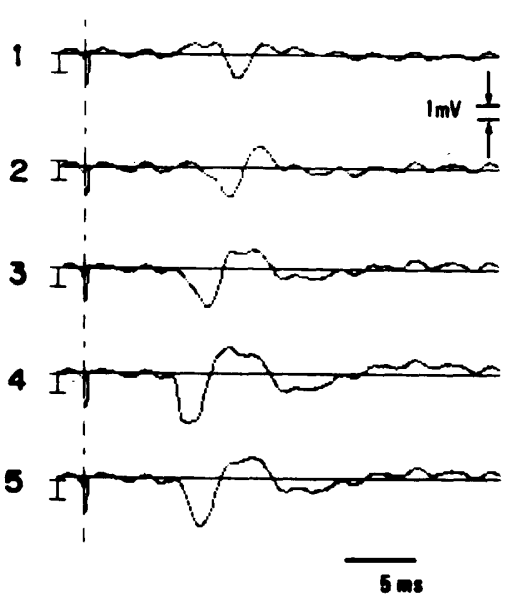

Fig. 4. A: schematic illustration of flexor pollicis brevis (FPB) muscle activated by stimulating the innervating motor axon (St.) with a tungsten microelectrode. Twelve surface electrodes were used for recording action potentials. In reality, stimulation was applied at the level of the elbow joint rather than close to the muscle as shown. B: action potentials recorded from surface electrodes. Number at the left of each action potential coincides with the counterpart in A. Calibrations are shown in the figure and upward deflection means positivity.

2. Stimulation of a single motor axon and UDLT of the frog muscle fiber

A single motor axon innervating the frog sartorius muscle was stimulated and the muscle action potential was extracellularly recorded with sixteen electrodes. Latency of action potentials varied systematically depending on the position of the recording electrode. The reddish tint shows the negativity of the potential, while blue shows positivity in this case. The deeper the tint, the larger the amplitude. As shown in Fig. 3E, the potential indicated by red was distributed on a convex curve. This means that the action potential first appeared at electrodes 8 and 9, and was then conducted bi-directionally from the plateau portion. Since the widths and slopes of the red lines in both directions were the same, it was concluded that the recorded action potentials were a result of conduction along the same single muscle fiber, and that the stimulated motor axon had synaptic contact (end-plate) with the muscle fiber between electrodes 8 and 9. The slope of the red line was linear, indicating that the conduction velocity was constant. From the slope of the red line in Fig. 3E, the conduction velocity of the action potential was measured to be $1.9 \mathrm{~m} / \mathrm{s}$. 


\section{DISCUSSION}

Bi-dimensional topographic analysis has been applied to muscle evoked potentials such as $\mathrm{H}$ - or $\mathrm{T}$ waves in the triceps surae muscle in man (Homma et al., 1981), and the travelling of $\mathrm{H}$ - and $\mathrm{T}$ waves was observed. In the present study, to determine the conduction velocity of the action potential in human muscle fibers, muscle fibers were electrically stimulated through the skin and over the muscle and bi-dimensional topography was calculated. Although the stimulus intensity was adjusted to elicit only a small fascicular twitch contraction of the muscle, more muscle fibers are activated by this stimulation than by stimulation of the motor axon through a tungsten microelectrode. If the excitability of muscle fibers depends on their fiber diameter, the action potentials recorded in the present study were contributed by muscle fibers with different fiber diameters. In all muscles examined, multiphasic compound action potentials were recorded. For example, in Fig. 3A, B, and $\mathrm{C}$, several blue lines do not run in parallel, which means that the action potentials that comprise the multiphasic compound potential are conducting at different speeds. Since the potential which appeared at first may distort the wave of the succeeding potentials, only the first blue line was analyzed to obtain the muscle conduction velocity.

STÅLberg (1966), using a single fiber EMG electrode, reported conduction velocities for different muscle fibers in man: for $\mathrm{m}$. frontalis, $2.0 \pm 0.4 \mathrm{~m} / \mathrm{s}$; for $\mathrm{m}$. extensor digitorum communis, $3.15 \pm 0.8 \mathrm{~m} / \mathrm{s}$; for m. biceps brachii, $3.39 \pm 0.7 \mathrm{~m} / \mathrm{s}$. Buchthal et al. (1955), using three concentric needle electrodes inserted with a mutual distance of $15-35 \mathrm{~mm}$ along the fiber direction, measured the conduction velocity for $\mathrm{m}$. brachialis as $4.72 \pm 0.54 \mathrm{~m} / \mathrm{s}$. These values were obtained from a single EMG during voluntary contraction of the muscle, and were calculated from a very minute time difference and electrode distance. On the other hand, using spike potentials recorded from surface electrodes during a weak voluntary contraction of m. vastus medialis, MoRimoto et al. (1980) measured the conduction velocity of the muscle to be $3.5 \mathrm{~m} / \mathrm{s}$. In our present study, the conduction velocity for three different muscles was measured from the slopes of the equipotential lines in UDLT. We believe that the conduction velocities measured over longer distances and listed in Table 1 are much more reliable than those previously reported.

Motor axons were stimulated with a tungsten microelectrode prepared for a microneurogram. By moving the electrode manually inside the median nerve and adjusting the stimulus intensity, a very small, weak contraction of the muscle was elicited. Since the threshold to elicit a small twitch contraction was less than $0.5 \mathrm{~V}$ (duration, $0.2 \mathrm{~ms}$ ), it is likely that only a few motor axons with a similar diameter or a single motor axon were activated by the stimulus. Therefore, action potentials recorded from the surface electrodes seem to be the sum of action potentials elicited in muscle fibers that belong in the same functional category or to the same motor unit. Since we obtained a linear relationship between latency and electrode position in the UDLT, conduction velocity of an action potential along each muscle fiber 
seemed to be uniform.

As seen in Fig. 3D and E, the equipotential line followed a convex-like pattern. When Fig. 3D and E are compared with an inverted Fig. 2 of Morimoto et al. (1980), these figures have a quite similar pattern. This means that an action potential first appeared at the plateau portion, and was then conducted bidirectionally from these. Since the width and slope of the lines were the same in both directions, it was assumed that the stimulated motor axon had synaptic contact (end-plate) with the muscle fiber at the plateau. Thus, it is possible to detect the endplate region on a muscle fiber in man, using a tungsten microelectrode to stimulate a single motor axon.

\section{REFERENCES}

Buchthal, F., Guld, C., and Rosenfalck, P. (1955) Innervation zone and propagation velocity in human muscle. Acta Physiol. Scand., 35: 174-190.

Homma, S., Iwata, K., Kusama, T., and NaKajima, Y. (1983a) Effects of electrical constants on conduction velocity of action potentials measured with unidimensional latency-topography in frog skeletal muscle fibers. Jpn. J. Physiol., 33: 711-720.

Homma, S., Kotaki, H., Mizote, M., Nakajima, Y., and Tamura, Z. (1984) Saltatory conduction of peripheral nerve impulse in clioquinol-treated rats. Neurosci. Lett., 45: 259-265.

Homma, S., Mizote, M., and NaKajima, Y. (1983b) Saltatory conduction revealed by unidimensional latency-topography of peripheral nerve impulse. Neurosci. Lett., 39: 225-230.

Homma, S., Nakajima, Y., Hayashi, K., Shito, M., and Sato, K. (1981) Area display of H, $\mathrm{M}$ and $\mathrm{T}$ waves. In: Muscle Receptors and Movement, ed. by TAYLOR, $\mathrm{A}$. and Prochazka, A., Macmillan Publ. Ltd., London, pp. 381-387.

HuXleY, A. F. and STÄMPFLI, R. (1949) Evidence for saltatory conduction in peripheral myelinated nerve fibers. J. Physiol. (Lond.), 108: 315-339.

Morimoto, S., Umazume, Y., and Masuda, M. (1980) Properties of spike potentials detected by a surface electrode in intact human muscle. Jpn. J. Physiol., 30: 71-80.

RASMinSKy, M. and SeARS, T. A. (1972) Internodal conduction in undissected myelinated nerve fibers. J. Physiol. (Lond.), 227: 323-350.

STÅLberG, E. (1966) Propagation velocity in human muscle fibers in situ. Acta Physiol. Scand. (Suppl. 287), 70: 1-112. 